\title{
A glycolytic product is obligatory for initiation of the sperm acrosome reaction and whiplash motility required for fertilization in the mouse
}

\author{
Lynn R. Fraser and P. J. Quinn* \\ Departments of Human Biology and *Biochemistry, Chelsea College, University of London, \\ London SW3 6LX, U.K.
}

\begin{abstract}
Summary. The substrate requirements for capacitation of epididymal mouse spermatozoa, initiation of the acrosome reaction and support of fertilization of mouse eggs in vitro were examined by assessing not only fertilization rates, but also the stages of egg activation and sperm head decondensation in fertilized eggs to monitor the temporal aspects of these processes. Although early events of capacitation did not require exogenous substrates, the fertilization process was effectively blocked at the terminal stages of capacitation in the absence of a glycolysable sugar, and addition of glucose was obligatory to initiate both the acrosome reaction and the whiplash motility associated with fertilizing ability. Once the spermatozoa had been primed by glucose, however, the removal of exogenous glucose did not block fertilization. The need for oxidative metabolism was excluded because successful fertilization could be achieved with glucose as the sole exogenous substrate under strictly anaerobic conditions or in the presence of $2.5 \mu \mathrm{M}$-oligomycin. We suggest, therefore, that epididymal mouse spermatozoa can be almost completely capacitated in the absence of metabolic processes simply by release into substrate-free medium. They require exposure to glucose to permit induction of the acrosome reaction and motility changes, necessary prerequisites for fertilization; such exposure is followed by rapid and synchronous penetration of eggs.
\end{abstract}

\section{Introduction}

Despite the fact that fructose is by far the most abundant hexose substrate in the semen of a wide variety of mammalian species (Mann, 1964), it does not appear to support capacitation and fertilization in those species so far examined. Fertilization was not obtained in in-vitro systems of mouse (Hoppe, 1976) and rat (Niwa \& Iritani, 1978) gametes when the sole exogenous substrate was fructose, although when glucose was present the rate was high. Most culture media employed for in-vitro fertilization and preimplantation embryonic development contain three potential exogenous substrates, i.e. pyruvate, lactate and glucose. It was originally suggested that all three substrates were required to support fertilization in the mouse (Miyamoto \& Chang, 1973) but lactate is now known to be inessential (Hoppe, 1976). No systematic studies have been reported, however, to examine critically the substrate requirements for capacitation and fertilization.

The development of systems capable of capacitating spermatozoa and supporting fertilization in vitro greatly facilitates studies of exogenous substrate requirements for these 
processes, and Fraser (1979a, b) has described techniques capable of distinguishing temporal events associated with capacitation and the acrosome reaction, which is a necessary prerequisite for penetration of the egg investments prior to fusion with the egg plasma membranes. We have now exploited the mouse system to examine the role of individual substrate requirements for capacitation, the acrosome reaction and fertilization and, more specifically, to define the relative importance of glycolytic and oxidative metabolism in these processes.

\section{Materials and Methods}

\section{Fertilization in vitro}

The basic medium used throughout these studies was a modified Tyrode's solution (Whittingham, 1971; Fraser \& Drury, 1975). The complete medium contained $5.56 \mathrm{~mm}$ glucose, $25 \mathrm{~mm}$-lactate and $0.5 \mathrm{~mm}$-pyruvate, as well as bovine serum albumin $(30 \mathrm{mg} / \mathrm{ml}$ : Armour, Eastbourne, Sussex). When variations in medium composition involved omission of lactate, additional $\mathrm{NaCl}$ was included to maintain osmolarity; no such compensation was considered necessary with the omission of glucose or pyruvate. All fine biochemicals were obtained from Sigma (St Louis, Missouri) unless stated otherwise.

The in-vitro fertilization system was essentially that described by Fraser \& Drury (1975). Egg donors were outbred TO females induced to superovulate with injections of 7.5 i.u. PMSG (Gestyl: Organon, Morden, Surrey) and 5 i.u. hCG (Pregnyl: Organon); unfertilized eggs were recovered $14 \mathrm{~h}$ after hCG injection. TO males, 2 per experiment, were used to provide epididymal sperm suspensions. In experiments involving several media of different composition, at least 3 media, including one known to permit high levels of fertilization, were examined in individual experiments. All suspensions contained gametes from both donors to eliminate variations between donors. After preincubation for $2 \mathrm{~h}$ at $37^{\circ} \mathrm{C}$, sperm suspensions were diluted 10 -fold in the appropriate medium to give a final concentration of $2 \times 10^{6} \mathrm{cells} / \mathrm{ml}$, eggs surrounded by cumulus cells were released from oviducts directly into suspensions and the gametes were incubated at $37^{\circ} \mathrm{C}$. Except for experiments using anaerobic conditions, the gas phase for gamete incubations was $5 \% \mathrm{CO}_{2}+5 \% \mathrm{O}_{2}+90 \% \mathrm{~N}_{2}$ or $5 \% \mathrm{CO}_{2}$ in air. No differences were observed between these 2 gas mixtures and the experimental results have been combined. All sperm suspensions were examined after the $2 \mathrm{~h}$ preincubation and dilution for assessment of sperm motility patterns (Fraser, 1977). Comparisons were made between spermatozoa in control medium and those maintained in the test media containing no or only selected exogenous substrates.

\section{Assessment}

Approximately $65 \mathrm{~min}$ after gamete mixing, eggs were removed from sperm suspensions and transferred to a small droplet of sperm-free medium under paraffin oil. All eggs were fixed by addition of excess neutral phosphate-buffered formalin $75 \mathrm{~min}$ after introduction to sperm suspensions. Eggs were subsequently stained with $0.5 \%$ aceto-orcein and examined microscopically for resumption of meiosis II (activation) and sperm head decondensation. Each fertilized egg was assessed for stage of egg activation (early anaphase, middle-late anaphase and telophase-second polar body) and for degree of sperm head decondensation (1, just beginning; 2, half-completed; and 3, completed) (Fraser, 1979a).

Determinations were also made of the presence or absence of acrosomes on spermatozoa preincubated for $2 \mathrm{~h}$ in various test media. In view of the well-established correlation between motility and fertilizing ability, the samples were enriched for motile gametes by applying several drops of the suspensions to short column beds $(2 \mathrm{~cm})$ of Sephadex G-25, prepared in Pasteur pipettes plugged with cotton wool, and eluting with the appropriate medium. Because the 
mouse sperm acrosome is difficult to observe in a motile cell, it was then necessary to immobilize the gametes before assessment. This was accomplished by mixing one drop of suspension with one drop of $0.01 \%$ aqueous acridine orange which acted immediately.

For each sample, two preparations were examined with visible light since the acridine orange was not being used for its fluorescent properties, and 70-100 spermatozoa in each were assessed for the presence of an acrosome.

\section{Individual substrates}

In the first series of experiments, preincubation and fertilization were carried out in media containing a single exogenous substrate: $5.56 \mathrm{~mm}$-glucose, $25.0 \mathrm{~mm}$-lactate, $0.5 \mathrm{~mm}$-pyruvate or $12 \mathrm{~mm}$-pyruvate. The last was included to provide an energy yield similar to the first two, assuming utilization of the tricarboxylic acid cycle.

Because these initial experiments indicated a requirement for glucose to obtain fertilization, the non-metabolizable glucose analogue 3-O-methylglucoside was tested on the assumption that any events initiated by glucose would be mimicked by the analogue. After preincubation for $2 \mathrm{~h}$ in the presence of $5.56 \mathrm{~mm}-3-\mathrm{O}$-methylglucoside, fertilization was examined in medium containing the glucoside or $5.56 \mathrm{~mm}$-glucose. Additionally, 2-deoxyglucose was also examined; this deoxysugar is phosphorylated in a reaction mediated by hexokinase to 2-deoxyglucose-6phosphate which then acts as a potent inhibitor of phosphoglucose isomerase and hence glycolytic metabolism. To determine the temporal involvement of glucose, preincubation for $2 \mathrm{~h}$ was carried out in media containing no or only a single substrate, with a glucose-containing medium used for fertilization. To examine the effect of glucose removal after a $2 \mathrm{~h}$ preincubation in glucose-containing medium, sperm suspensions were washed by centrifuging at $800 \mathrm{~g}$ for $4 \mathrm{~min}$ and re-suspending in glucose-free medium. This procedure was repeated, the re-suspended suspension was diluted as described above in glucose-free medium and eggs then added.

\section{Inhibition of oxidative metabolism}

To examine the fertilizing capacity of gametes under conditions which inhibited oxidative metabolism, sperm suspensions were preincubated in media with and without glucose, then diluted into glucose-containing medium for fertilization. Replicate samples were maintained under aerobic and anaerobic $\left(5 \% \mathrm{CO}_{2}+95 \% \mathrm{~N}_{2}\right)$ conditions. In the latter case, all manipulations, including release of spermatozoa, dilution and release of eggs, were carried out as rapidly as possible under paraffin oil equilibrated with the appropriate gas mixture.

The effect of oligomycin, an inhibitor of oxidative phosphorylation, was examined by preincubating sperm suspensions in medium containing glucose and $2.5 \mu \mathrm{M}$-oligomycin; suspensions were then washed as described above to remove excess oligomycin. To verify the oligomycin-induced inhibition of oxidative phosphorylation, oxygen uptake was measured polarographically with an oxygen electrode maintained at $37^{\circ} \mathrm{C}$. Uptake was measured first in the presence of $5.56 \mathrm{~mm}$-glucose alone and then after addition of sufficient oligomycin to give the same final concentration used for in-vitro fertilization studies $(2 \cdot 5 \mu \mathrm{M})$.

\section{Alternative metabolic pathways}

The ability of $5.56 \mathrm{~mm}$-fructose to substitute for glucose was examined by comparing this substrate with very low concentrations of glucose $(0.56 \mathrm{mM})$. In a further series of experiments, an attempt was made to divert fructose into metabolic reactions of the Hers' pathway (Sillero, Sillero \& Sols, 1969), the major metabolic pathway of fructose in the liver. This is characterized by the entry of fructose into the glycolytic pathway at the level of triose phosphate rather than hexokinase. The diversion utilized inclusion of 2-deoxyglucose, to block the phosphoglucose isomerase, and fructose. 
The other catabolic pathway that glucose is likely to enter is the hexose monophosphate shunt. There are no known specific inhibitors of this pathway, but one of the products that might be required for fertilization is NADPH. This reduced nucleotide was tested at concentrations of 2 and $10 \mathrm{~mm}$ together with $5 \mathrm{~mm}$-pyruvate as an energy substrate. Since spermatozoa have been shown to contain malic enzyme (Mounib, 1974), endogenous generation of NADPH was examined by incubating sperm suspensions in medium containing pyruvate and introducing 5 mM-malate and 2 mM-NADP ${ }^{+}$when eggs were added.

Finally, 5 mm exogenous ATP was tested. Unlike other reaction products, however, there was no way to generate intracellular ATP by alternative reactions and the uptake of the ATP by spermatozoa could not be verified under the conditions utilized.

\section{Results}

\section{Individual substrates}

The general reliability of this in-vitro fertilization system was demonstrated when the medium contained all 3 exogenous substrates or glucose plus pyruvate (Table 1). Fertilization rates were very high and advanced stages of egg activation and sperm head decondensation predominated, suggesting that sperm penetration had occurred shortly after mixing of gametes (Fraser, 1979a). Neither pyruvate nor lactate alone was capable of supporting fertilization. To eliminate the possibility of slower penetration under these conditions, in one experiment eggs were incubated for $6 \mathrm{~h}$ but still proved to be unfertilized. When glucose alone was included, fertilization rates and stages of nuclear development were indistinguishable from those in medium containing 2 or 3 of the substrates, indicating a need for glycolytic metabolism.

Table 1. Fertilization in vitro obtained with mouse sperm suspensions preincubated for $2 \mathrm{~h}$ in medium containing a single exogenous energy source $(G=5.56$ mM-glucose; $L=25$ mM-lactate; $P=0.5$ mm-pyruvate; High $\mathrm{P}=12 \mathrm{mM}$-pyruvate) and diluted into medium of the same composition

\begin{tabular}{|c|c|c|c|c|c|c|c|c|}
\hline \multirow[b]{2}{*}{ Energy source } & \multirow[b]{2}{*}{$\begin{array}{l}\text { No. of } \\
\text { exps }\end{array}$} & \multirow[b]{2}{*}{$\begin{array}{c}\text { Eggs } \\
\text { fertilized* }\end{array}$} & \multicolumn{3}{|c|}{ Egg stage $(\%)$} & \multicolumn{3}{|c|}{$\begin{array}{c}\text { Sperm head staget, } \\
\%\end{array}$} \\
\hline & & & $\begin{array}{c}\text { Early } \\
\text { anaphase }\end{array}$ & $\begin{array}{l}\text { Mid-late } \\
\text { anaphase }\end{array}$ & $\begin{array}{l}\text { Telophase-2nd } \\
\text { polar body }\end{array}$ & 1 & 2 & 3 \\
\hline $\begin{array}{l}G+P \text { or } \\
G+L+P\end{array}$ & 4 & $\begin{array}{c}38 / 39 \\
(97.4 \%)\end{array}$ & 0 & $\begin{array}{l}1 \\
(2 \cdot 6)\end{array}$ & $\begin{array}{l}37 \\
(97 \cdot 4)\end{array}$ & 0 & 2.5 & 97.5 \\
\hline L & 3 & $\begin{array}{c}0 / 29 \\
(0 \%)\end{array}$ & & & & & & \\
\hline $\mathbf{p}$ & 3 & $\begin{array}{l}0 / 37 \\
(0 \%)\end{array}$ & & & & & & \\
\hline High P & 3 & $\begin{array}{r}0 / 37 \\
(0 \%)\end{array}$ & & & & & & \\
\hline G & 3 & $\begin{array}{c}37 / 38 \\
(97 \cdot 3 \%)\end{array}$ & 0 & 0 & $\begin{array}{c}37 \\
(100)\end{array}$ & 0 & $2 \cdot 3$ & $97 \cdot 7$ \\
\hline
\end{tabular}

* By 75 min after gamete mixing.

+ Of those fertilized: see text for detailed description of stages.

No fertilization was observed with 3-O-methylglucoside $(0 / 25)$, but the introduction of glucose at the time of gamete mixing resulted in successful fertilization (8/9) and advanced nuclear stages, again suggesting a requirement for glycolysis rather than simply the presence of the glucose molecule. Inclusion of 2-deoxyglucose, with or without glucose in the preincubation medium, whether or not it was present in the fertilization medium containing glucose, prevented fertilization $(0 / 30)$. General motility was markedly reduced in the presence of 2-deoxyglucose, consistent with the expected inhibition of glycolysis by this compound and the resulting reduction in available energy sources to support motility. 
The temporal involvement of glucose in the fertilization process was ascertained by preincubation of spermatozoa in various media lacking glucose, with the introduction of glucose at the time of gamete mixing. Regardless of the composition of the initial medium, the addition of glucose immediately conferred fertilizing ability on the spermatozoa, as evidenced by the high fertilization rates and advanced stages of egg activation and sperm head decondensation (Table 2). These results were indistinguishable from those obtained with complete medium, suggesting that glucose is only required during the terminal stages of capacitation, i.e. just before the acrosome reaction (see below).

When glucose was removed from spermatozoa preincubated in the presence of glucose by washing and resuspending in glucose-free medium, no reduction in fertilization rates per se or in the rate of penetration was observed (Table 3).

Table 2. Fertilization in vitro obtained with mouse sperm suspensions preincubated for $2 \mathrm{~h}$ in medium containing a single exogenous energy source and diluted into medium containing glucose and pyruvate

( $\mathrm{G}=5.56 \mathrm{~mm}$-glucose; $\mathrm{L}=25 \mathrm{~mm}$-lactate; $\mathrm{P}=0.5 \mathrm{~mm}$-pyruvate; High $\mathrm{P}=12 \mathrm{~mm}$-pyruvate)

\begin{tabular}{|c|c|c|c|c|c|c|c|c|c|}
\hline \multirow{2}{*}{\multicolumn{2}{|c|}{ Medium for: }} & \multirow{3}{*}{$\begin{array}{l}\text { No. of } \\
\text { exps }\end{array}$} & \multirow{3}{*}{$\begin{array}{c}\text { Eggs } \\
\text { fertilized* }\end{array}$} & \multicolumn{3}{|c|}{ Egg stage $+(\%)$} & \multirow{2}{*}{\multicolumn{3}{|c|}{$\begin{array}{l}\text { Sperm head } \\
\text { staget, \% }\end{array}$}} \\
\hline & & & & \multirow{2}{*}{$\begin{array}{c}\text { Early } \\
\text { anaphase }\end{array}$} & \multirow{2}{*}{$\begin{array}{l}\text { Mid-late } \\
\text { anaphase }\end{array}$} & \multirow{2}{*}{$\begin{array}{l}\text { Telophase- } \\
\text { 2nd polar body }\end{array}$} & & & \\
\hline Preincubation & n Fertilization & & & & & & 1 & 2 & 3 \\
\hline $\mathbf{G}+\mathbf{L}+\mathbf{P}$ & $G+L+P$ & 3 & $\begin{array}{c}47 / 50 \\
(94.0 \%)\end{array}$ & 0 & 0 & $\begin{array}{c}47 \\
(100)\end{array}$ & $4 \cdot 1$ & $6 \cdot 1$ & $89 \cdot 8$ \\
\hline$G+P$ & $G+P$ & 8 & $\begin{array}{l}184 / 200 \\
(92 \cdot 0 \%)\end{array}$ & 0 & $\begin{array}{c}3 \\
(1 \cdot 6)\end{array}$ & $\begin{array}{l}181 \\
(98 \cdot 2)\end{array}$ & 1.7 & $6 \cdot 1$ & $92 \cdot 2$ \\
\hline L & $G+P$ & 3 & $\begin{array}{c}60 / 69 \\
(86.9 \%)\end{array}$ & 0 & 0 & $\begin{array}{c}60 \\
(100)\end{array}$ & $4 \cdot 1$ & 1.4 & 94.6 \\
\hline $\mathbf{P}$ & $G+P$ & 3 & $\begin{array}{c}69 / 77 \\
(89.6 \%)\end{array}$ & 0 & 0 & $\begin{array}{c}69 \\
(100)\end{array}$ & $1 \cdot 3$ & $8 \cdot 0$ & $90 \cdot 7$ \\
\hline High P & $G+P$ & 4 & $\begin{array}{r}93 / 105 \\
(88.6 \%)\end{array}$ & 0 & $\begin{array}{l}2 \\
(2 \cdot 2)\end{array}$ & $\begin{array}{c}91 \\
(97 \cdot 8)\end{array}$ & $1 \cdot 1$ & $6 \cdot 6$ & $92 \cdot 3$ \\
\hline G & $G+P$ & 3 & $\begin{array}{c}66 / 76 \\
(86.8 \%)\end{array}$ & 0 & 0 & $\begin{array}{c}66 \\
(100)\end{array}$ & $2 \cdot 1$ & $4 \cdot 3$ & $93 \cdot 6$ \\
\hline- & $G+P$ & 3 & $\begin{array}{c}47 / 52 \\
(88.7 \%)\end{array}$ & 0 & $\begin{array}{c}1 \\
(2 \cdot 1)\end{array}$ & $\begin{array}{c}46 \\
(97.9)\end{array}$ & 0 & $12 \cdot 5$ & $87 \cdot 5$ \\
\hline
\end{tabular}

* By 75 min after gamete mixing.

$\dagger$ Of those fertilized: see text for detailed description of stages.

Table 3. Fertilization in vitro obtained with mouse sperm suspensions preincubated for $2 \mathrm{~h}$ in $5.56 \mathrm{mM}$ glucose $(G)$, washed in glucose-free medium, and diluted in glucose-free medium

\begin{tabular}{|c|c|c|c|c|c|c|c|c|c|}
\hline \multicolumn{2}{|c|}{ Medium for: } & \multirow[b]{2}{*}{$\begin{array}{l}\text { No. of } \\
\text { exps }\end{array}$} & \multirow[b]{2}{*}{$\begin{array}{c}\text { Eggs } \\
\text { fertilized* }\end{array}$} & \multicolumn{3}{|c|}{ Egg stage $+(\%)$} & \multicolumn{3}{|c|}{$\begin{array}{l}\text { Sperm head } \\
\text { staget, \% }\end{array}$} \\
\hline Preincubation & Fertilization & & & $\begin{array}{c}\text { Early } \\
\text { anaphase }\end{array}$ & $\begin{array}{l}\text { Mid-late } \\
\text { anaphase }\end{array}$ & $\begin{array}{l}\text { Telophase- } \\
\text { 2nd polar body }\end{array}$ & 1 & 2 & 3 \\
\hline $\mathrm{G}$ & $\mathrm{G}$ & 3 & $\begin{array}{c}48 / 53 \\
(90 \cdot 6 \%)\end{array}$ & 0 & $\begin{array}{l}1 \\
(2 \cdot 1)\end{array}$ & $\begin{array}{l}47 \\
(97.9)\end{array}$ & $6 \cdot 0$ & $6 \cdot 0$ & 88.0 \\
\hline $\mathrm{G}$ & -.. & 3 & $\begin{array}{c}45 / 51 \\
(88 \cdot 2 \%)\end{array}$ & 0 & 0 & $\begin{array}{c}45 \\
(100)\end{array}$ & 4.4 & 0 & 95.6 \\
\hline
\end{tabular}

* By 75 min after gamete mixing.

$\dagger$ Of those fertilized: see text for detailed description of stages.

\section{Inhibition of oxidative metabolism}

Under aerobic conditions, fertilization rates were high and advanced nuclear stages predominated whether glucose was present throughout or added at the time of sperm dilution 
(Table 4). Under anaerobic conditions, fertilization rates were slightly lower in both groups and earlier stages of egg activation and sperm head decondensation were noted in the majority of eggs, although several observations suggested the latter to be due primarily to alterations in the responses of the eggs. In many eggs the chromosomes appeared abnormally fuzzy and there was often evidence of a loss of spindle integrity with stray chromosomes visible. Moreover, unusual combinations of egg and sperm nuclear stages were noted, such as eggs at middle anaphase with sperm heads at stages 2 and 3, rather than the usual stage 1 (Fraser, 1979b). Similarly, stage 3 heads, rather than stages 1 and 2, were frequently seen in eggs at late anaphase. Most unusual were the 2 eggs at early anaphase, one with a sperm head at stage 2 and the other one at stage 3 . However, glycolytic metabolism was again shown to be sufficient to support fertilization.

Table 4. A comparison of the fertilization obtained in vitro under conditions which inhibit oxidative metabolism

\begin{tabular}{|c|c|c|c|c|c|c|c|c|c|}
\hline \multirow{2}{*}{\multicolumn{2}{|c|}{ Medium for: }} & \multirow{3}{*}{$\begin{array}{l}\text { No. of } \\
\text { exps }\end{array}$} & \multirow{3}{*}{$\begin{array}{c}\text { Eggs } \\
\text { fertilized }\end{array}$} & \multicolumn{3}{|c|}{ Egg stage* $(\%)$} & \multirow{2}{*}{\multicolumn{3}{|c|}{$\begin{array}{l}\text { Sperm head } \\
\text { stage*, } \%\end{array}$}} \\
\hline & & & & & & & & & \\
\hline Preincubation & Fertilization & & & anaphase & anaphase & 2 nd polar body & 1 & 2 & 3 \\
\hline \multicolumn{10}{|l|}{ Aerobic } \\
\hline G & G & 3 & $\begin{array}{c}41 / 41 \\
(100 \%)\end{array}$ & $\begin{array}{l}2 \\
(4 \cdot 9)\end{array}$ & $\begin{array}{c}6 \\
(14 \cdot 6)\end{array}$ & $\begin{array}{c}33 \\
(80 \cdot 5)\end{array}$ & $4 \cdot 5$ & $13 \cdot 6$ & $81 \cdot 8$ \\
\hline- & $G$ & 3 & $\begin{array}{c}44 / 47 \\
(93.6 \%)\end{array}$ & $\begin{array}{l}2 \\
(4 \cdot 5)\end{array}$ & $\begin{array}{c}12 \\
(27 \cdot 2)\end{array}$ & $\begin{array}{c}30 \\
(68 \cdot 2)\end{array}$ & $22 \cdot 7$ & 11.4 & $65 \cdot 9$ \\
\hline $\begin{array}{l}\text { Anaerobic } \\
\mathrm{G}\end{array}$ & $G$ & 3 & $\begin{array}{c}51 / 63 \\
(81.0 \%)\end{array}$ & $\begin{array}{l}15 \\
(29 \cdot 4)\end{array}$ & $\begin{array}{l}21 \\
(41 \cdot 1)\end{array}$ & $\begin{array}{l}15 \\
(29 \cdot 4)\end{array}$ & $68 \cdot 4$ & $22 \cdot 8$ & $8 \cdot 8$ \\
\hline - & $\mathrm{G}$ & 3 & $\begin{array}{c}61 / 77 \\
(79.2 \%)\end{array}$ & $\begin{array}{l}16 \\
(26 \cdot 2)\end{array}$ & $\begin{array}{c}21 \\
(34.4)\end{array}$ & $\begin{array}{l}24 \\
(39 \cdot 3)\end{array}$ & $51 \cdot 6$ & $19 \cdot 4$ & $30 \cdot 6$ \\
\hline \multicolumn{10}{|l|}{$\begin{array}{l}\text { Oligomycin } \\
\qquad(2.5 \mu \mathrm{M})\end{array}$} \\
\hline G & G & 3 & $\begin{array}{c}63 / 63 \\
(100 \%)\end{array}$ & 0 & $\begin{array}{l}1 \\
(1 \cdot 6)\end{array}$ & $\begin{array}{l}62 \\
(98 \cdot 4)\end{array}$ & $3 \cdot 0$ & 1.5 & $95 \cdot 5$ \\
\hline $\begin{array}{l}\mathrm{G}+\text { oligo- } \\
\text { mycin } \\
(2 \cdot 5 \mu \mathrm{M})\end{array}$ & G & 3 & $\begin{array}{c}56 / 56 \\
(100 \%)\end{array}$ & 0 & $\begin{array}{l}2 \\
(3 \cdot 6)\end{array}$ & $\begin{array}{l}54 \\
(96.4)\end{array}$ & 1.8 & 1.8 & $96 \cdot 5$ \\
\hline
\end{tabular}

* Of those fertilized: see text for detailed description of stages.

$\uparrow$ Mouse sperm suspensions were preincubated for $2 \mathrm{~h}$ under the appropriate conditions; when necessary glucose (G. $5.56 \mathrm{mM}$ ) was introduced at the time of gamete mixing. All eggs were fixed after $75 \mathrm{~min}$.

The failure of $2.5 \mu \mathrm{M}$-oligomycin to inhibit fertilization (Table 4) was consistent with this interpretation. Washing the spermatozoa proved necessary to prevent deleterious effects of inhibition of oxidative phosphorylation in the egg, but this procedure is unlikely to relieve inhibition of the relevant processes in spermatozoa since oligomycin is assumed to bind irreversibly to the mitochondrial ATPase enzyme. The concentration of oligomycin used was considered sufficient to inhibit oxidative phosphorylation completely on the basis of experiments measuring oxygen uptake by sperm suspensions. In a typical experiment, oxygen uptake of washed mouse spermatozoa increased from 7.5 to $9.8 \mathrm{nmol} \mathrm{O}_{2}$ per $10^{8}$ cells $/ \mathrm{min}$ when glucose was added to a final concentration of $5.56 \mathrm{~mm}$. Subsequent addition of oligomycin to a final concentration of $2.5 \mu \mathrm{M}$ reduced oxygen uptake to $5.3 \mathrm{nmol} \mathrm{O}_{2}$ per $10^{8} \mathrm{cells} / \mathrm{min}$, and higher concentrations of oligomycin did not reduce this further. These measurements therefore suggest that oxidation is tightly coupled to phosphorylation in these cells and that the oligomycin was sufficient to inhibit oxidative metabolism via the tricarboxylic acid cycle. 


\section{Alternative metabolic pathways}

When fructose rather than glucose was included as the sole exogenous source, no fertilization was obtained (Table 5), although introduction of glucose with the eggs permitted rapid fertilization and penetration rates similar to those obtained with glucose present throughout. Preincubation in exogenous substrate-free medium and dilution into medium containing 0.56 mm-glucose, i.e. one-tenth the usual concentration, produced fertility patterns not markedly different from the control incubations with $5.56 \mathrm{~mm}$ glucose throughout. Attempts to divert fructose into the Hers' pathway by including 2-deoxyglucose resulted in no fertilization $(0 / 10)$, suggesting that products of this pathway, if formed, were not required. The reduced nucleotide NADPH, which is a product of the hexose monophosphate shunt, proved unable to support fertilization at 2 or $10 \mathrm{~mm}$, in combination with $5 \mathrm{~mm}$-pyruvate $(0 / 43)$. Because exogenous nucleotide might fail to penetrate the gamete, attempts were made to induce endogenous production of the NADPH via malic enzyme-mediated reduction of $\mathrm{NADP}^{+}$but this also gave negative results $(0 / 27)$. Finally, exogenous ATP, supplied in the absence of glucose, proved unable to support fertilization $(0 / 23)$, but the inability to generate ATP intracellularly via alternative reactions does not permit exclusion of this glycolytic product from consideration as a possible product involved in fertilization.

Table 5. Fertilization in vitro obtained with mouse sperm suspensions preincubated for $2 \mathrm{~h}$ in fructose and diluted into medium containing fructose $(F=5.56 \mathrm{~mm})$ or glucose $\left(\mathrm{G}=5.56 \mathrm{~mm}, \mathrm{G}^{*}=0.56 \mathrm{~mm}\right)$

\begin{tabular}{|c|c|c|c|c|c|c|c|c|c|}
\hline \multirow{2}{*}{\multicolumn{2}{|c|}{ Medium for: }} & \multirow{3}{*}{$\begin{array}{l}\text { No. of } \\
\text { exps }\end{array}$} & \multirow{3}{*}{$\begin{array}{c}\text { Eggs } \\
\text { fertilized* }\end{array}$} & \multicolumn{3}{|c|}{ Egg stage $(\%)$} & \multirow{2}{*}{\multicolumn{3}{|c|}{$\begin{array}{c}\text { Sperm head } \\
\text { staget, } \%\end{array}$}} \\
\hline & & & & & & & & & \\
\hline Preincubation & Fertilization & & & anaphase & anaphase & 2nd polar body & 1 & 2 & 3 \\
\hline G & $\mathrm{G}$ & 3 & $\begin{array}{c}33 / 34 \\
(97 \cdot 1 \%)\end{array}$ & 0 & 0 & $\begin{array}{c}33 \\
(100)\end{array}$ & 5.7 & $2 \cdot 9$ & 91.4 \\
\hline $\mathrm{F}$ & $\mathrm{F}$ & 3 & $0 / 32$ & & & & & & \\
\hline $\mathrm{F}$ & G & 3 & $\begin{array}{c}28 / 30 \\
(93 \cdot 3 \%)\end{array}$ & 0 & 0 & $\begin{array}{c}28 \\
(100)\end{array}$ & $3 \cdot 4$ & 6.9 & $89 \cdot 7$ \\
\hline - & $\mathrm{G}^{*}$ & 3 & $\begin{array}{c}26 / 29 \\
(89.6 \%)\end{array}$ & 0 & 0 & $\begin{array}{c}26 \\
(100)\end{array}$ & $11 \cdot 5$ & $7 \cdot 7$ & $80 \cdot 8$ \\
\hline
\end{tabular}

* By 75 min after gamete mixing.

† Of those fertilized: see text for detailed description of stages.

\section{Sperm motility patterns}

The motility pattern of the spermatozoa was strikingly correlated with their fertilizing ability. In all media s"pahle of supporting fertilization, i.e. all those containing glucose, the majority of single moti!? spermatozoa exhibited a characteristic whiplash motility pattern. In contrast, spermatozoa in all media lacking glucose exhibited a typical progressive, albeit sluggish, motility pattern associated with non-fertilizing cells (Fraser, 1977). On addition of glucose to preincubated suspensions whiplash motility patterns could be detected within a few minutes and the level of activity generally increased. When spermatozoa, preincubated in glucose-containing medium, were washed and resuspended in glucose-free medium, a whiplash pattern was observed initially but gradually disappeared over a 30 -min period.

\section{Acrosome loss}

As the results in Table 6 clearly indicate, only a small proportion (about 10\%) of spermatozoa were found to lack an acrosome when preincubated for $2 \mathrm{~h}$ in the absence of glucose, in contrast to the much higher figure of approximately $40 \%$ in the presence of glucose. Within 
a few minutes of the introduction of glucose, the proportion of spermatozoa without acrosomes had increased to about $40 \%$. Consistent with the fertilization levels presented in Table 4 , the presence of $2.5 \mu \mathrm{M}$-oligomycin did not interfere with acrosome loss. We would not expect the gel filtration technique to bias the selection of cells in groups on any characteristic other than motility and there was no indication that a higher proportion of cells were non-motile in media lacking glucose, i.e. the same relative proportion of motile gametes was present in each group. Furthermore, earlier observations on motile cells after a similar incubation time indicated that mouse spermatozoa lacking acrosomes were still highly motile.

Table 6. Proportion of mouse spermatozoa lacking an acrosome after preincubation for $2 \mathrm{~h}$ in media containing $5.56 \mathrm{~mm}$-glucose $(\mathrm{G}), 12 \mathrm{~mm}$ pyruvate $(\mathrm{P})$ or no exogenous substrate

\begin{tabular}{|c|c|c|c|}
\hline \multicolumn{2}{|c|}{ Medium for: } & \multirow{2}{*}{$\begin{array}{l}\text { No. lacking acrosomes/ } \\
\text { total examined }\end{array}$} & \multirow[b]{2}{*}{ Mean \pm s.d. } \\
\hline Preincubation & Assessment & & \\
\hline G & G & $510 / 1282$ & $0.40 \pm 0.03$ \\
\hline- & G & $109 / 259$ & $0.42 \pm 0.04$ \\
\hline $\mathbf{P}$ & $\mathbf{P}$ & $84 / 595$ & $0.14 \pm 0.02$ \\
\hline $\mathrm{P}$ & G & $223 / 566$ & $0.39 \pm 0.03$ \\
\hline- & - & $15 / 257$ & $0.06 \pm 0.02$ \\
\hline $\mathrm{G}+$ oligomycin $(2.5 \mu \mathrm{M})$ & $\mathrm{G}+$ oligomycin $(2.5 \mu \mathrm{M})$ & $183 / 499$ & $0.37 \pm 0.03$ \\
\hline
\end{tabular}

\section{Discussion}

The results clearly show that of the three substrates normally present in culture media used for capacitation and fertilization in in-vitro studies, only glucose is necessary in the murine system. There is no fertilization if pyruvate or lactate is the only exogenous substrate supplied or if a glucose analogue or an inhibitor of glycolytic metabolism is introduced. A requirement for glucose has also been inferred from studies of rat spermatozoa reported by Niwa \& Iritani (1978), who showed that preincubation of cells in the absence of glucose failed to support fertilization unless glucose was added. Because assessment of eggs revealed earlier stages of development than those obtained when glucose was present throughout it was suggested that the sugar was in some way involved in capacitation. The fact that other studies have indicated an obligatory requirement for pyruvate and/or lactate to achieve fertilization (mouse: Miyamoto \& Chang, 1973; rat: Tsunoda \& Chang, 1975) may simply represent an effect on the viability of eggs rather than the absolute fertilizing ability of spermatozoa. For example, in circumstances in which prolonged incubation of the spermatozoa and eggs is required to achieve fertilization, failure of the eggs to promote normal nuclear development in media lacking pyruvate does not necessarily reflect deficiencies in the spermatozoa. Hoppe (1976) has reported pyruvate to be necessary for cleavage of fertilized mouse eggs and we have been able to confirm this by continuing a few cultures beyond the usual termination point of $75 \mathrm{~min}$ used in the present experiments.

The in-vitro fertilization technique described here has been successfully used to establish the temporal requirements for glucose during the capacitation and fertilization processes in the mouse. Capacitation is a time-dependent process which permits spermatozoa to undergo the acrosome reaction, a necessary prerequisite to successful fertilization; although this phenomenon is common to many mammalian species, the length of time required for capacitation varies according to species (Bedford, 1970). We consider a population of spermatozoa to be fully capacitated if it demonstrates both rapid and synchronous fertilization of eggs (see Tables 1-5); in the mouse the period required to meet these conditions is approximately $2 \mathrm{~h}$ (Fraser \& Drury, 1976; Fraser, 1979a, b). The failure to obtain fertilization under experimental 
conditions (see Tables 1 and 5) does not necessarily indicate that spermatozoa are totally uncapacitated, but only that they have failed to complete the full sequence of events; within the temporal framework of capacitation they may be partly or even almost totally capacitated. Because significant changes in sperm fertilizing ability, acrosomes and motility patterns were observed within a few minutes of glucose introduction, the simplest interpretation of our results is that some product of glycolysis is required during the terminal phase of capacitation to initiate the acrosome reaction and whiplash motility. Spermatozoa preincubated in the absence of exogenous substrates for sufficient time to permit full capacitation in complete medium (Fraser \& Drury, 1976) were unable to fertilize eggs until supplied with glucose; fertilization then took place immediately and nuclear development advanced to stages indistinguishable from those obtained with glucose present throughout the preincubation and gamete mixing periods. This response of sperm suspensions to such manipulation is quite distinct from that observed when spermatozoa preincubated in glucose-containing medium for the much shorter period of 30 min were mixed with eggs. Under the latter conditions, penetration was asynchronous as evidenced by the spread in stages of nuclear development with few eggs possessing terminal stages. Therefore, although a few spermatozoa may be fully capacitated after $30 \mathrm{~min}$, the majority of cells are not and the population of spermatozoa does not exhibit the rapid and synchronous penetration obtained in the present experiments with a 2 -h preincubation. Further evidence that spermatozoa can become almost fully capacitated in the complete absence of metabolic activity is provided by the high fertilization rates obtained with cells preincubated under anaerobic conditions in the absence of a glycolysable sugar; release of the spermatozoa into substrate-free medium is all that appears to be required. The fact that eggs fertilized under anaerobic conditions did not achieve the advanced stages of nuclear development observed in those incubated under aerobic conditions appeared to be due, at least in part, to an effect on the egg as detailed earlier in the results section.

Evidence for a glycolytic requirement to permit induction of the acrosome reaction is presented in Table 6; the results clearly indicate that, in the absence of glucose, only a few spermatozoa (approximately 10\%) had lost the acrosome and these may well represent dead cells. In the presence of glucose, whether continuous or added just before assessment, a sizeable proportion of cells (approximately 40\%) had no acrosome. Although the method used for assessment screened a whole population of spermatozoa, the results were very similar to those obtained with selected individual motile spermatozoa (36\% lacking acrosomes; Fraser, 1977). Furthermore, the correlation between high rates of acrosome loss and demonstrably high fertilization rates after the addition of glucose (Table 2) suggests that the induction of the acrosome reaction associated with glycolytic metabolism is a physiological rather than a "false" process as described by Bedford (1970). It would seem probable, however, that only spermatozoa undergoing the acrosome reaction in close proximity to the egg can achieve successful fertilization.

It is also evident that glycolytic metabolism is required to support the development of the whiplash motility pattern associated with fertilizing ability in mouse (Fraser, 1977), hamster (Yanagimachi, 1970) and guinea-pig (Yanagimachi, 1972) spermatozoa. Although fertilization did not occur in the absence of this characteristic motility pattern, any medium which promoted motility changes also promoted induction of the acrosome reaction, and at present it is not possible to evaluate the relative contribution of these two events to successful fertilization.

While glucose has a demonstrated role in priming spermatozoa for fertilization, our studies suggest that exogenous sugar is not required during actual penetration of the egg investments or fusion of the spermatozoon with the egg. Removal of exogenous glucose from the preincubated spermatozoa by a washing procedure capable of reducing initial components by more than two orders of magnitude (L. R. Fraser, unpublished observation) did not reduce the fertilizing ability of the spermatozoa. In all experiments, cumulus cells were present on eggs at the time of mixing with spermatozoa, yet fertilization failed when no exogenous glucose was provided. From this we conclude that cumulus cells do not, in themselves, provide a factor or 
factors sufficient to support fertilization in the absence of glucose. Furthermore, it has proved possible to achieve rapid fertilization of cumulus-free eggs in medium containing only glucose (L. R. Fraser, unpublished observations).

The inability of a fructose metabolite to replace one derived from glucose is difficult to explain, especially since Hoppe (1976) has demonstrated that mouse spermatozoa can metabolize fructose. Our results are, however, consistent with previous observations that fructose cannot support fertilization in vitro (Hoppe, 1976; Niwa \& Iritani, 1978). Metabolism of fructose by spermatozoa has always been considered to be via glycolysis, but possible diversion into the Hers' pathway (Sillero et al., 1969) has not been critically examined. Although there is some evidence from the pattern of radiolabelled glycerol metabolism (Brown-Woodman, Mohri, Mohri, Suter \& White, 1978) that at least some enzymic steps of the Hers' pathway operate in spermatozoa, we were unable to obtain fertilization when fructose metabolism was diverted into this route. We were also unable to demonstrate any specific requirement for the hexose monophosphate shunt, a result consistent with that of Hoppe (1976) who was unable to detect significant metabolism of glucose along that route.

The pivotal role of glycolysis in fertilization also explains the antifertility action of $\alpha$ chlorohydrin. A metabolite of the drug irreversibly inhibits glyceraldehyde-3-phosphate dehydrogenase (Brown-Woodman et al., 1978) and, thus, based on our results, presumably prevents spermatozoa from undergoing the acrosome reaction; it also has a demonstrated effect on motility. Since the drug is an effective antifertility agent in a variety of mammalian species, it is likely that glycolysis may be a general requirement for initiation of the acrosome reaction in capacitated spermatozoa. A report to the contrary is that of Rogers \& Yanagimachi (1975), who showed that induction of the acrosome reaction in guinea-pig spermatozoa was more rapid and extensive in media containing pyruvate and lactate than in media containing glucose, whether alone or in combination with the other two. Moreover, oligomycin and inhibitors of electron transport were found to prevent spermatozoa undergoing the acrosome reaction with consequent reduction in fertility (Rogers, Ueno \& Yanagimachi, 1977). These results are in direct contrast to those obtained with mouse gametes. In a study of hamster spermatozoa (Bavister \& Yanagimachi, 1977) it was reported that glucose played a supporting rather than a primary role in induction of the acrosome reaction.

Our results clearly indicate an obligatory requirement for glycolytic metabolism of glucose to permit induction of the acrosome reaction and whiplash motility; although abundant in seminal fluid, fructose is incapable of filling this role. The practical implication of these results is that ejaculated spermatozoa cannot proceed beyond the terminal stages of capacitation without a supply of glucose from female tract secretions.

\section{References}

Bavister, B.D. \& Yanagimachi, R. (1977) The effects of sperm extracts and energy sources on the motility and acrosome reaction of hamster spermatozoa in vitro. Biol. Reprod. 16, 228-237.

Bedford, J.M. (1970) Sperm capacitation and fertilization in mammals. Biol. Reprod. 2, Suppl. 2, 128158.

Brown-Woodman, P.D.C., Mohri, H., Mohri, T., Suter, D. \& White, I.G. (1978) Mode of action of $\alpha$ chlorohydrin as a male anti-fertility agent. Biochem. $J$. 170, 23-37.

Fraser, L.R. (1977) Motility patterns in mouse spermatozoa before and after capacitation. J. exp. Zool. 202 , 439-444.

Fraser, L.R. (1979a) Rate of fertilization in vitro and subsequent nuclear development as a function of the post-ovulatory age of the mouse egg. J. Reprod. Fert. $55,153-160$.
Fraser, L.R. (1979b) Accelerated mouse sperm penetration in vitro in the presence of caffeine. J. Reprod. Fert. 57, 377-384.

Fraser, L.R. \& Drury, L.M. (1975) The relationship between sperm concentration and fertilization in vitro of mouse eggs. Biol. Reprod. 13, 513-518.

Fraser, L.R. \& Drury, L.M. (1976) Mouse sperm genotype and the rate of egg penetration in vitro. J. exp. Zool. 197, 13-20.

Hoppe, P.C. (1976) Glucose requirement for mouse sperm capacitation in vitro. Biol. Reprod. 15 , $39-45$.

Mann, T. (1964) The Biochemistry of Semen and the Male Reproductive Tract. Methuen, London.

Miyamoto, H. \& Chang, M.C. (1973) The importance of serum albumin and metabolic intermediates for capacitation of spermatozoa and fertilization of mouse eggs in vitro. J. Reprod. Fert. 32. 193-205. 
Mounib, M.S. (1974) NAD- and NADP-malic enzymes in spermatozoa of mammals and fish. FEBS Letters 48, 79-84.

Niwa, K. \& Iritani, A. (1978) Effect of various hexoses on sperm capacitation and penetration of rat eggs in vitro. J. Reprod. Fert. 53, 267-271.

Rogers, B.J. \& Yanagimachi, R. (1975) Retardation of guinea pig sperm acrosome reaction by glucose: the possible importance of pyruvate and lactate metabolism in capacitation and the acrosome reaction. Biol. Reprod. 13, 568-575.

Rogers, B.J., Ueno, M. \& Yanagimachi, R. (1977) Inhibition of hamster sperm acrosome reaction and fertilization by oligomycin, antimycin $\mathrm{A}$ and rotenone. J. exp. Zool. 199, 129-136.
Sillero, M.A.G., Sillero, A. \& Sols, A. (1969) Enzymes involved in fructose metabolism in liver and the glyceraldehyde metabolic crossroads. Eur. J. Biochem. 10, 345-350.

Tsunoda, Y. \& Chang, M.C. (1975) In vitro fertilization of rat and mouse eggs by ejaculated sperm and the effect of energy sources on in vitro fertilization of rat eggs. J. exp. Zool. 193, 79-86.

Whittingham, D.G. (1971) Culture of mouse ova. J. Reprod. Fert., Suppl. 14, 7-21.

Yanagimachi, R. (1970) The movement of golden hamster spermatozoa before and after capacitation. J. Reprod. Fert. 23, 193-196.

Yanagimachi, R. (1972) Fertilization of guinea pig eggs in vitro. Anat. Rec. 174, 9-20.

Received 18 January 1980 